\title{
La Route de la Carpe frite dans le Sundgau : enjeu identitaire, enjeu territorial
}

Die touristische Straße des frittierten Karpfens : Thema für Identität, Thema für Raumordnung

\section{Raymond Woessner}

\section{OpenEdition}

\section{Journals}

Electronic version

URL: http://journals.openedition.org/rge/5786

ISSN: 2108-6478

\section{Publisher}

Association des géographes de l'Est

Printed version

Date of publication: 22 April 2016

ISSN: 0035-3213

\section{Electronic reference}

Raymond Woessner, «La Route de la Carpe frite dans le Sundgau : enjeu identitaire, enjeu territorial », Revue Géographique de l'Est [Online], vol.56 / n $1-2$ | 2016, Online since 03 May 2017, connection on 08 September 2020. URL : http://journals.openedition.org/rge/5786

This text was automatically generated on 8 September 2020

Tous droits réservés 


\title{
La Route de la Carpe frite dans le Sundgau : enjeu identitaire, enjeu territorial
}

\author{
Die touristische Straße des frittierten Karpfens : Thema für Identität, Thema für \\ Raumordnung
}

Raymond Woessner

1 Mal connus, les territoires de marge offrent pourtant des spécificités qui les rendent particulièrement intéressants dans leurs dynamiques de développement (Woessner, 2010). Le Sundgau entre dans la problématique des marges par bien des aspects. Pour le Dictionnaire d'analyse spatiale, "marginal équivaut vite à accessoire, délaissé, dévitalisé, à territoire-musée » (Bavoux, Chapelon, 2014, cf. p. 362). S'il est facile de décrire ainsi le Sundgau, l'enjeu de cet article se situe dans la suite de la définition : "Moins soumises aux contrôles sociaux, bénéficiant de normes moins contraignantes et de davantage de souplesse, plus libres en somme, les marges peuvent faire preuve de plus d'inventivité. [..] Les marges, franges de contact entre systèmes, peuvent ainsi fonctionner comme des interfaces créatives, organiser leur vie propre, en particulier s'il y a perméabilité frontalière et tendance à l'intégration réciproque de deux marges accolées » (id, cf. p. 363). Leur problème est alors d'établir un jeu d'acteurs spécifique qui permette la création d'un système localisé, usuellement en associant des forces exogènes comme endogènes sur la base d'une créativité enracinée dans le terroir. Dans le cas du Sundgau, il n'est pas certain que les forces vives présentes puissent y parvenir, ni qu'elles arrivent à récupérer de l'énergie dans les centres extérieurs de Bâle, Mulhouse et Belfort-Montbéliard.

2 Le Sundgau compte parmi l'une de sept grandes régions d'étangs françaises avec la Brenne, la Sologne, la Champagne, la Lorraine, les Dombes et le Forez (Bartout, 2006). L'objectif de cet article est de voir jusqu'à quel point les étangs des bords de l'Ill et de la Largue servent de support à la carpiculture, qui a elle-même permis la création de la Route de la Carpe frite, et vers quoi cette ébauche de territorialisation peut désormais tendre à la faveur de l'étalement urbain et de la simplification du mille-feuille 
administratif. Pourra-t-on évoquer un «territoire limnique » (Bartout, Touchart, 2015), à la fois unité paysagère et fonctionnelle, et porteuse d'un projet fondée sur un jeu d'acteurs porté par le monde scientifique, politique, associatif et économique?

Placés en dérivation, ou bien établis en chapelet dans des vallons adjacents jusqu'à se perdre dans les bois, les étangs font partie du patrimoine paysager sundgauvien depuis des siècles. Mais ils n'apparaissent ni dans les recueils de son poète Nathan Katz (1892-1981), ni dans les œuvres picturales recensées par l'Association Art de HauteAlsace. Il en va de même pour la carpe en général. Alors que sa rivale "la truite va joyeuse le long des bords fleuris " dans le Lied de Schubert, elle est un poisson dont l'image est souvent négative, car elle se nourrit de vase et elle ne se montre guère aux promeneurs. Et Amélie Nothomb ne laisse aucune chance à cyprinus carpo : «Je ne sais pas si vous avez déjà vu la bouche d'une carpe en train de se nourrir mais je ne connais pas de spectacle aussi dégueulasse $»^{1}$.

4 Pourtant, dans le Sundgau, les étangs et leurs hôtes sont des marqueurs d'identité et même des éléments de branding territorial comme le montre la Route de la Carpe frite. Cet article se propose donc de remettre le sujet dans son contexte et de voir jusqu'à quel point on peut constater une forme d'endogénéisation locale. Il n'a pas d'autre ambition que le cadre monographique et un sujet plus vaste serait de comparer les formes de promotion économique observables dans d'autres contrées d'étangs, en France comme dans les régions germaniques.

\section{Le Sundgau, une marge territoriale avec un « pays d'étangs »}

5 Dans l'espace du Rhin supérieur, qui est riche de son armature urbaine et de ses campagnes peuplées, il existe des angles morts à la ruralité avérée, soit en plaine lorsque les forêts alluviales sont restées prégnantes, soit dans des espaces bordiers de collines comme celui du Sundgau. Ce petit pays, étymologiquement « la région du sud » et dont les limites sont floues, est bloqué entre les Vosges et le Jura, barré par la ligne de partage des eaux entre la mer du Nord et la Méditerranée, cerné par Mulhouse, Bâle, Montbéliard et Belfort. Il est traversé, un peu au nord, par l'autoroute A36, la ligne de chemin de fer historique empruntée par les TGV et le vieux canal du Rhône-au-Rhin à l'usage désormais improbable (Figure 1, 3 et 7).

6 Hier comme aujourd'hui, les douces ondulations des collines jouent un rôle important. Les villages et leurs extensions se construisent d'abord dans les talwegs, malgré le risque dû aux inondations le long de l'ill, de la Largue ou de leurs modestes affluents. "Ce qui semble spécifique du Sundgau semble aller en s'intensifiant jusqu'à atteindre son paroxysme au lieu même où il disparaît, quelque part du côté des sources de l'Ill et de la Largue, cédant la place à l'Ajoie, ou si l'on veut, à ce qu'il est convenu d'appeler aujourd'hui le Jura alsacien » (Urban, 2010, cf. p. 493). En effet, en allant vers le sud, les hêtraies sont progressivement remplacées par des conifères et les forêts impressionnent par leurs dimensions relatives. L'humidité est prégnante, notamment parce que les précipitations de la Trouée de Belfort sont abondantes (900-1.200 mm/an) et que les placages de loess périglaciaires se gonflent volontiers des eaux de pluie avant de les restituer progressivement. Il en résulte un monde un peu clos, replié sur luimême, où des traditions de sorcellerie ont laissé la place à de nombreux guérisseurs aujourd'hui encore. Avant 1914, les citadins se moquaient des paysans sundgauviens 
qui « ne se déplacent pas plus loin que de l'étable au fumier et retour. Dont l'horizon est bouché. Ce en quoi ils n'avaient certainement pas tort. Notre mère, par exemple, n'était jamais allée plus loin qu'au marché d'Altkirch ", écrit Achille Walch (2016).

Dans les inventaires dressés par G. Bischoff (2007) pour 1850 et par L.G. Werner (1920) à la parution de son article, la vallée de la Largue compte 120 étangs répartis sur 120 ha, celle de l'Ill 70 étangs sur 100 hectares. La plupart des étangs sont de petite taille; dans le Territoire de Belfort, 62 \% d'entre eux ont moins de 50 ares (Kolar, 1991). La mise en eau de nouveaux étangs s'est poursuivie depuis. Ainsi, à Carspach, l'étang du Himmelreich date de 1932 (Glotz, 2006, cf. p. 133) ; à Zillisheim, l'étang de pêche du Bielsberg date de la fin des années 1980. C'est pourquoi le Haut-Sundgau a pu être qualifié de "Petite Sologne" "avec ses quelque cinq cents étangs» répartis dans " deux cent cinquante $\mathrm{km}^{2}$ environ » et il « appartient aux grandes régions françaises à étangs " (Schortanner, 1978, cf. p.14). Pour la Maison de la nature du Sundgau, ces étangs sont aujourd'hui "plus de mille, dont les deux tiers sont situés dans la haute vallée de la Largue, entre Seppois et Mertzen" (brochure disponible dans son présentoir à Altenach, non-signée).

Les meilleurs étangs se situent dans les terres de culture dont le bassin-versant « draine une eau plus fertile en minéraux et moins acide qu'une eau qui vient de la forêt » avec en conséquence une meilleure croissance des carpes; il faut environ 3 ans pour faire un poisson de $1 \mathrm{~kg}$ et on peut compter 300 carpes par hectare d'étang (Haennig, 1974, cf. p. 117). Le dispositif est complété par de petits étangs de village, des carpiers ou carpières. Ces bassins reçoivent des poissons en automne, ou bien avant d'être mis en vente ; ils sont alimentés par une eau courante qui retarde ainsi la formation de glace en surface. Enfin, dans les années 1920 encore, Werner évoque les derniers chasseurs de grenouilles professionnels, très actifs autour des étangs.

9 La problématique de la biodiversité nous intéresse ici non en tant que telle, mais dans l'appréciation de sa subjectivité selon les auteurs. M. Schortanner (1978) évoque des nénuphars en pleine eau, et, sur les rives, des roselières qui cèdent progressivement devant des aulnes et autres arbustes; des frênes viennent en transition face à la hêtraie ; dans le détail, « deux étangs voisins peuvent être totalement différents par la faune et la flore » (Schortanner, id., p. 18). Mais cette nature a largement disparu au profit de l'agriculture productiviste qui la cerne, traditionnellement avec la mise en herbe et plus récemment avec la maïsiculture. Finalement, la biodiversité des étangs serait « extrêmement faible $»^{2}$. Leurs berges abruptes en ont fait des étangs baignoires, peu profonds, au niveau d'eau constant et de taille modeste (Figure 1). Les étangs sundgauviens ne sont donc plus des écosystèmes mais des sociaux-systèmes. 


\section{CARPE FRITE DU SUNDGAU (à la semoule)}

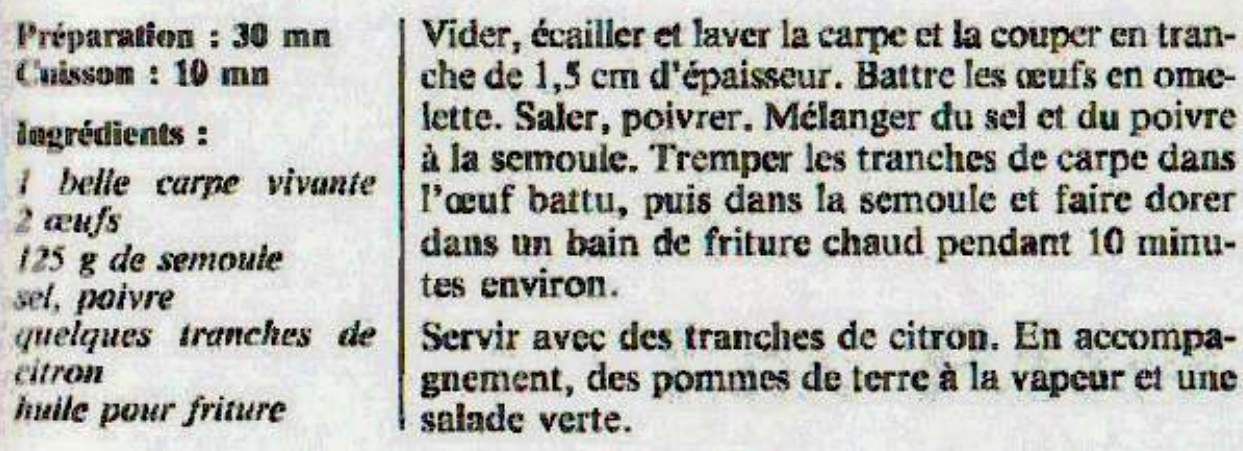

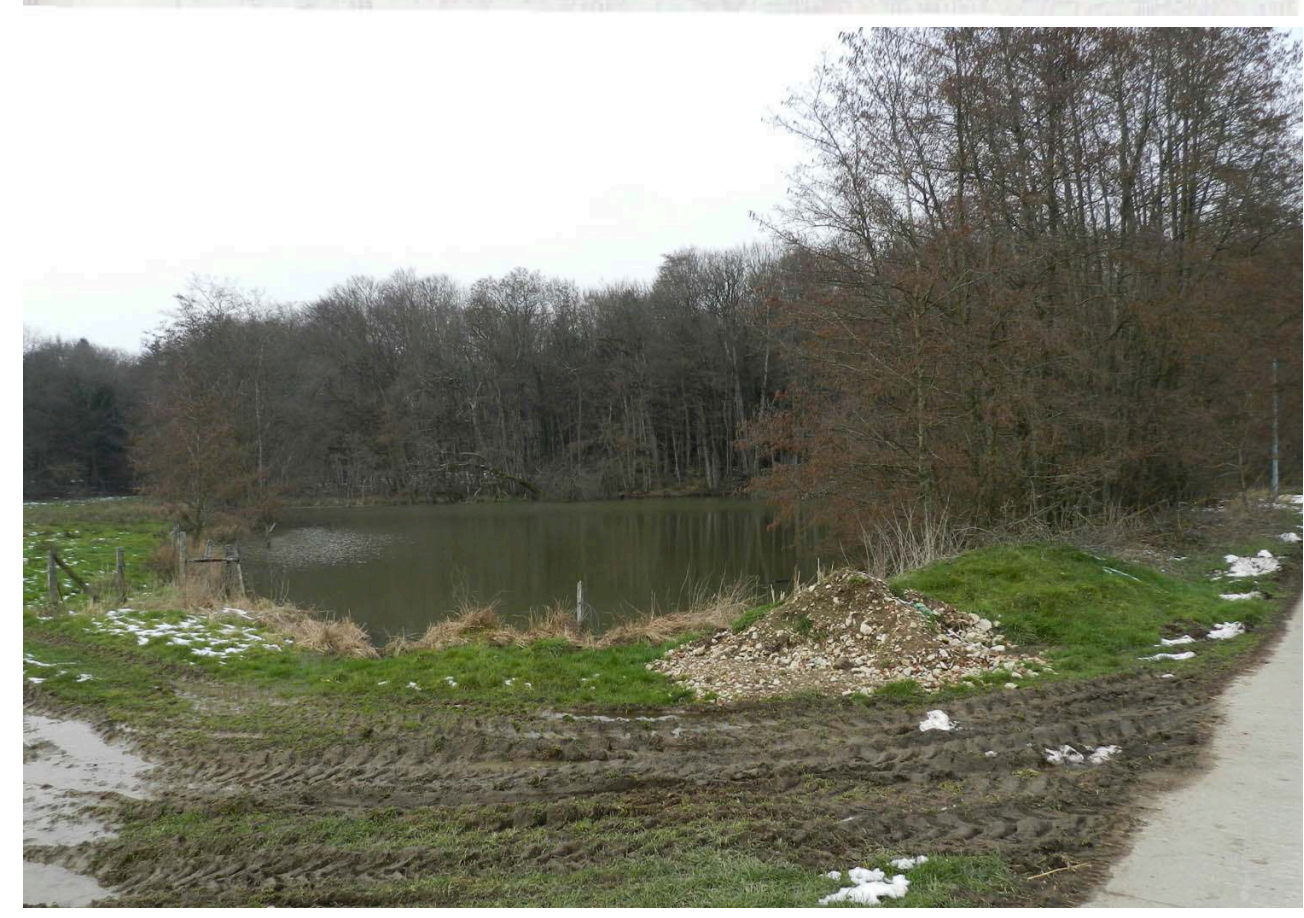

10 Aux étangs Nérac d'Altenach-Saint-Ulrich, le Conseil général du Haut-Rhin a soutenu le rêve d'un retour à une situation «naturelle » idéale (mais laquelle ?) en y promouvant la renaturation à partir de 2003; on y bague chaque année des oiseaux migrateurs. Selon la Ligue de Protection des Oiseaux, les étangs du Sundgau occidental " constituent des relais importants pour les migrateurs: chevaliers (combattant, aboyeur, sylvain, culblanc,...), bécasseaux (variable, minute), bécassine des marais et parfois sourde, petit et grand gravelot. De nombreuses espèces sont nicheuses et migratrices à la fois, comme le bruant des roseaux, les rousserolles effarvatte et verderolle, l'hypolaïs polyglotte, la bergeronnette printanière.... ${ }^{3}$. Ces oiseaux et leurs habitats sont quasi tous protégés par l'arrêté du 29 octobre 2009.

11 De fait, les étangs sont inscrits dans des démarches environnementalistes. Lancé en 1993, le programme national des ZNIEFF sert de « zonages pour étudier et éclairer », et par la suite celui des programmes Natura 2000 de l'Union européenne de «zonages pour administrer et pour agir » (Le Gléau, 1999). En 2004 est apparue une zone Natura 
2000 intitulée «Sundgau, région des étangs » (Figure 1). Répartis dans 14 communes, 198 ha sont concernés. Environ $40 \%$ de la couverture est faite de hêtraies, $35 \%$ d'étangs et de rivières. On y recense 8 espèces protégées (le papillon cuivré des marais, le triton crêté, le crapaud sonneur à ventre jaune, des chauve-souris de 3 types murin, et, dans le règne végétal, des dicranes et des fougères d'eau à quatre feuilles). Le Conservatoire des Sites Alsaciens basé à l'Écomusée d'Ungersheim (Haut-Rhin) est chargé de la gestion de la zone. "Reste à élaborer un cahier des charges spécifique permettant de garantir un partage équilibré entre les fonctions de protection et la production forestière et à recueillir l'accord des propriétaires. Un effort de pédagogie envers les propriétaires forestiers privés devra être engagé ", écrit le Conservatoire (Natura 2000, 2015). En filigrane pointe la question du sens à donner aux étangs : conservatoires naturels, pêche, base de loisirs familiale ou pisciculture?

\section{Un ancien système productif fondé sur la carpiculture}

12 La carpe commune est probablement originaire d'Asie mineure. Elle se subdivise en 1.500 espèces dans le monde. Dépourvue d'estomac, elle est omnivore, robuste, et les œufs éclosent facilement si les eaux ont une température de $22-24^{\circ} \mathrm{C}$. Sous $10^{\circ} \mathrm{C}$, elle hiberne. L'espèce est benthivore; le zoobenthos et le zooplancton lui fournissent l'essentiel de sa nourriture en milieu naturel. Comme la carpe fouille les fonds, elle accroît la turbidité de l'eau; en hiver, elle s'enfouit dans la vase. La carpiculture nécessite plusieurs étangs. Idéalement, en proportion, il faut deux étangs de ponte, deux étangs de géniteurs et quatre étangs d'alevinage (Ranson, 2003). L'assec estival ou bien pratiqué selon une périodicité de 5 à 10 ans - permet de lutter contre les parasitoses et les risques d'eutrophisation, voire de cultiver un peu d'avoine qui ne sera pas récoltée mais servira d'alimentation aux carpes. En novembre, « les propriétaires ouvrent les vannes des étangs pour laisser s'écouler l'eau et récupérer les poissons. [..] Ils ramassent avec de grands paniers plusieurs quintaux de carpes, brochets, tanches et autres sandres. Après nettoyage, les prises atterrissent dans de grandes cuves remplies d'eau. Les hommes qui on participé à la pêche ont chaussé de hautes bottes qui les protègent jusqu'aux fesses. Car le fond de l'étang est tapissé d'une boue gluante dans laquelle on s'enfonce de $50 \mathrm{~cm}$ ou plus » (Richert, 1991, cf. p. 43). La carpe atteint un poids de trois kilos au bout de quatre étés. Comme le prix des farines de poisson ne cesse d'augmenter, les éleveurs remplacent celles-ci par des aliments industriels (par ex. un mélange de foie de bœuf, de levure et de vitamines pour les juvéniles, puis des produits de la salmoniculture en phase de croissance), à moins qu'ils n'utilisent des mélanges moins coûteux de céréales (Ranson, 2003). L'utilisation d'engrais ou de fumure organique (fumier, fiente de volaille) favorise la flore aquatique et donc la nourriture en zooplancton.

13 De modestes fouilles archéologiques témoignent de l'existence d'étangs à l'époque romaine et peut-être même dès le néolithique, sans que l'on ne connaisse leur usage (Werner, 1920). Plusieurs villas romaines étaient établies à Friesen, Hirtzbach, Hirsingue et Fulleren (Zimmermann, 1982), c'est-à-dire à $40 \mathrm{~km}$ des villes de Kembs (Cambete et son pont sur le Rhin) et de Mandeure (Epomanduodurum et son amphithéâtre). Un petit centre d'étape s'était ainsi niché sur cette voie très fréquentée qui reliait Lyon au Rhin. 
Les étangs ont pris de l'importance au XII ${ }^{\mathrm{e}}$ siècle lorsque l'abbaye cistercienne de Lucelle, située au pied du Jura, aujourd'hui à la frontière suisse, avait développé la pisciculture ; sur la Largue, le prieuré de Saint-Ulrich avait participé au mouvement. Un apogée avait été atteint au XVI ${ }^{\mathrm{e}}$ siècle, lorsque les archiducs d'Autriche avaient encouragé les étangs princiers et que les "gentlemen-pisciculteurs " (Bischoff, 2007) spoliaient les communaux pour y pratiquer la pisciculture. En 1525, lors de la Guerre des Paysans, les villageois avaient dénoncé le fait ; à eux seuls, les Morimont-Belfort auraient alors tiré le quart de leurs revenus communaux de leurs 25.000 carpes et brochets. Transportées vivantes, les carpes se vendaient jusqu'à Colmar et Fribourg en Brisgau. Les profits étaient énormes car la carpiculture nécessitait peu de personnel permanent (Bischoff, 2007) ; et les travaux d'entretien étaient faits dans le cadre des cinq jours annuels de corvée (Zimmermann,1982). Tant que le catholicisme est resté une religion prégnante, le commerce des carpes a connu le succès à cause du carême et des autres jours de jeûne où la viande est interdite.

Aujourd'hui, la pisciculture est un secteur d'avenir du fait de l'épuisement des ressources halieutiques (INRA, 2007). En comptant les producteurs littoraux, la France compte 2.500 emplois piscicoles qui en font le leader européen de la truite et des alevins marins. La production nationale de carpes se fait en étang et représente 6.000 tonnes/an, à comparer aux 35.000 tonnes de truites. L'encadrement réglementaire préfère le principe de précaution aux logiques économiques. En effet, la Loi Pêche de 1984, la Directive Cadre européenne sur l'Eau (DCE) de 2000, et davantage encore la Loi sur l'Eau de 2006, qui a créé l'office National de l'Eau et des Milieux Aquatiques (ONEMA), ont pour priorité la préservation de la ressource aquatique. La DCE définit les conditions de référence d'un système écologique comme celui prévalant en l'absence ou la quasi-absence de perturbations anthropiques (Bouleau, Pont, 2014). Quant à l'ONEMA, il est «l'organisme technique français de référence sur la connaissance et la surveillance de l'état des eaux et sur le fonctionnement écologique des milieux aquatiques $»^{4}$; il est en outre responsable de la police des eaux. La Loi sur l'Eau et les Milieux Aquatiques (LEMA) de 2006 regroupe les associations agréées de pêche et de pisciculture en une fédération départementale (Article 90) chargée d'organiser et d'encadrer les activités, mais le législateur ne propose aucune incitation quant à leur développement éventuel. Pascal Bartout et Laurent Touchart (2015) vont jusqu'à évoquer « l'omnipotence de la démarche biologique chez les représentants du pouvoir, au détriment des hommes, alors que les étangs et les autres petits plans d'eau artificiels sont des objets créés par et pour l'homme ». Dès 1984, la loi Pêche avait freiné la création de nouveaux étangs, pratiquement arrêtée depuis 2000 en France (Bartout, 2006).

16 Il en résulte un fiasco économique. "Alors que la demande du marché augmente et malgré un savoir-faire reconnu, il n'y a pas eu de création d'entreprises piscicoles en France depuis 1997 en raison du manque de disponibilité de sites et des difficultés pour accroitre les exploitations piscicoles du fait de contraintes environnementales » (INRA, 2007, cf. p. 6). C'est pourquoi les importations de perches d'Ouganda et de Tanzanie ainsi que de pangasius du Vietnam viennent peupler les étals français. De plus, les centrales d'achat des grands distributeurs préfèrent ces approvisionnements globalisés. Ainsi donc « la filière française rend les armes » (INRA, 2007, cf. p. 13). Les pisciculteurs sundgauviens subissent en outre la concurrence des importations de poisson vivant venus par camion des pays d'Europe centrale et orientale. Seules de petites entreprises 
fortement ancrées dans leur territoire, appuyées par les collectivités locales et adossées à des formes de créativité culinaire, semblent à même de pouvoir survivre, à l'image des deux pisciculteurs de Friesen.

Dans ce village de la Largue, deux entreprises piscicoles apparaissent comme des références (Figure 2). Spécialisés dans la carpe, Bihl comme Kohler avaient débuté leur activité dans les années 1950/1960, avant de se tourner également vers d'autres poissons d'eau douce, truites et saumons, mais aussi brochets, sandres, perches, silures... Pour Bihl (10 à 19 salariés), la commercialisation se fait dans l'est de la France. Pour Kohler ( 3 à 5 salariés), la production est destinée au repeuplement des plans et des cours d'eau, avec des associations de pêche ou des particuliers comme clients. Leurs laboratoires vendent des carpes en filets ou en darnes, ainsi que des truites en filet. Dans le Territoire de Belfort, Beaume joue un rôle équivalent, mais elle est implantée en marge du Sundgau, au pied des Vosges, près des étangs du Malsaucy, connus par ailleurs pourle festival des Eurockéennes. Dans le Canton suisse du Jura, à Courtemaîche près de Porrentruy, Choulard date elle aussi des années 1950. Elle est désormais la seule du canton.

Enfin, VMC Pêche fabrique des hameçons depuis 1910, d'abord à Grandvillars, puis également à Morvillars depuis 1938. Les familles Viellard et Migeon appartiennent à une dynastie d'industriels qui comptent parmi les Hénokiens, un cercle réservé aux entreprises de plus de deux cent ans d'existence. En 2000, VMC Pêche a fusionné avec le leader mondial des leurres, Rapala (Finlande), et en 2010 elle a reçu le label national « Entreprise du patrimoine vivant ». Ce label récompense les entreprises françaises aux savoir-faire artisanaux et industriels d'excellence. Elle s'agrandit en 2015 et emploie 150 salariés. La propriété foncière était probablement liée aux besoins en bois pour les fonderies de la famille au XVII e siècle. Charles Viellard avait importé des machines à fabriquer des hameçons depuis Redditch en Angleterre. Mais aujourd'hui, VMC est un global player qui fabrique plus de 60.000 hameçons par jour, y compris à Batam (Indonésie). Les liens entre la société et son territoire se sont distendus et ne sont plus qu'un souvenir. 
Figure 2 : Les activités liées aux étangs

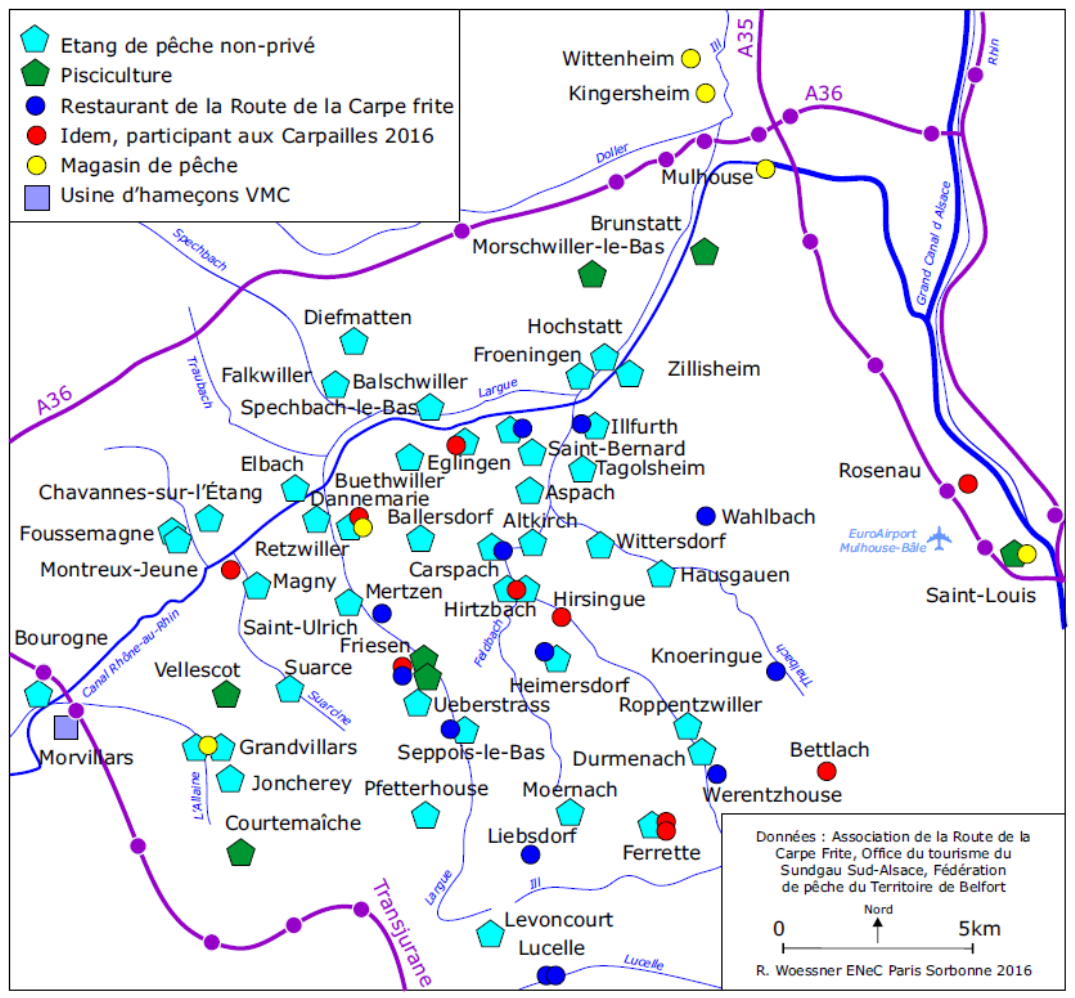

\section{La valorisation de l'héritage des étangs : la lente émergence d'un projet territorial}

Trois axes militent pour le développement d'un jeu d'acteurs portant un projet territorial où les étangs sont indispensables: la promotion de la gastronomie; la pêche ; la cohérence de l'action politique.

En 2010 est apparue l'association appelée Office de Tourisme du Sundgau, Sud Alsace, alors le premier constitué à l'échelle d'un Pays sous forme associative. Sa stratégie consiste à intégrer la carpe dans un tout où la pêche, la mémoire de 1914-1918, les véloroutes et le rapprochement avec les stratégies trinationales du Coin des Trois Frontières sont appelées à jouer un rôle important. L'Office a fort à faire quand on sait que le tourisme sundgauvien est une activité réduite à la portion congrue. En comparaison des Vosges et du vignoble alsacien, le Pays du Sundgau compte très peu $d$ 'hébergements, très peu d'investissements (au dernier rang d'Alsace) et presque pas de sites à entrées payantes (dernier rang là aussi) (ORTA, 2016). Apparu à Gommersdorf dans les années 1970, le mouvement de défense des maisons paysannes a débouché sur la création de l'Écomusée d'Alsace, mais celui-ci s'est implanté en 1984 à Ungersheim au nord de Mulhouse grâce au soutien actif du maire de cette commune. 


\title{
CARPE FRITE DU SUNDGAU (à la semoule)
}

\author{
Préparatien : $30 \mathrm{mn}$ \\ Cuissem : $10 \mathrm{mn}$ \\ Josgrédients : \\ 1 belle carne vivante \\ 2 aeu/s \\ $125 \mathrm{~g}$ de semoute \\ sel, poivre \\ quelques tranches de \\ citron \\ huile pour friture \\ Vider, écailler et laver la carpe et la couper en tran- \\ che de $1,5 \mathrm{~cm}$ d'épaisseur. Battre les nufs en ome- \\ lette. Saler, poivrer. Mélanger du sel et du poivre \\ à la semoule. Tremper les tranches de carpe dans \\ l'ceuf battu, puis dans la semoule et faire dorer \\ dans un bain de friture chaud pendant 10 minu- \\ tes environ. \\ Servir avec des tranches de citron. En accompa- \\ gnement, des pommes de terre à la vapeur et une \\ salade verte.
}

21 L'association Sundgau, Route de la Carpe frite, est apparue en 1975. Il ne s'agit pas d'une route bien définie que l'on pourrait suivre à l'image de la prestigieuse Route des Vins alsacienne, mais de restaurants qui se disséminent dans le Sundgau. C'est aux Journées d'Octobre de Mulhouse que l'idée avait pris corps entre des restaurateurs (dont Charles Ritter à Dannemarie) et la Chambre de Commerce et d'Industrie mulhousienne. Une trentaine de restaurateurs et pisciculteurs avaient alors reçu un large soutien départemental (Conseil général, Chambres professionnelles) et sundgauvien (syndicats hôteliers et piscicoles, maires), ainsi que de Tony Troxler, un folkloriste et auteur de théâtre alsacien, et des caves Wolfsberger dont le président était également le maire d'Éguisheim. Louis Heilmann, détaché de la $\mathrm{CCI}$, a été le premier secrétaire de l'association. La Route apparaît ainsi comme un bel exemple de réussite endogène, qui fédère les forces vives concernées et utilise Mulhouse pour sa promotion et son fonctionnement. Depuis, elle a planté son stand dans de nombreux salons régionaux ainsi qu'à Paris (au Salon Nature), à Berlin, Bruxelles... M. Reitzer, député-maire d'Altkirch, est président de l'association depuis 1987. La Route est inscrite aux Sites remarquables du Goût depuis 1992. Depuis 2005, chaque printemps, elle organise les Carpailles, où les restaurants participants proposent des recettes originales. En 2000, une délégation brésilienne a repris le concept de la Route pour le déployer dans la région de Nova Friburgo, une ville de tourisme et d'horticulture située dans l'arrière-pays montagneux de Rio de Janeiro. 


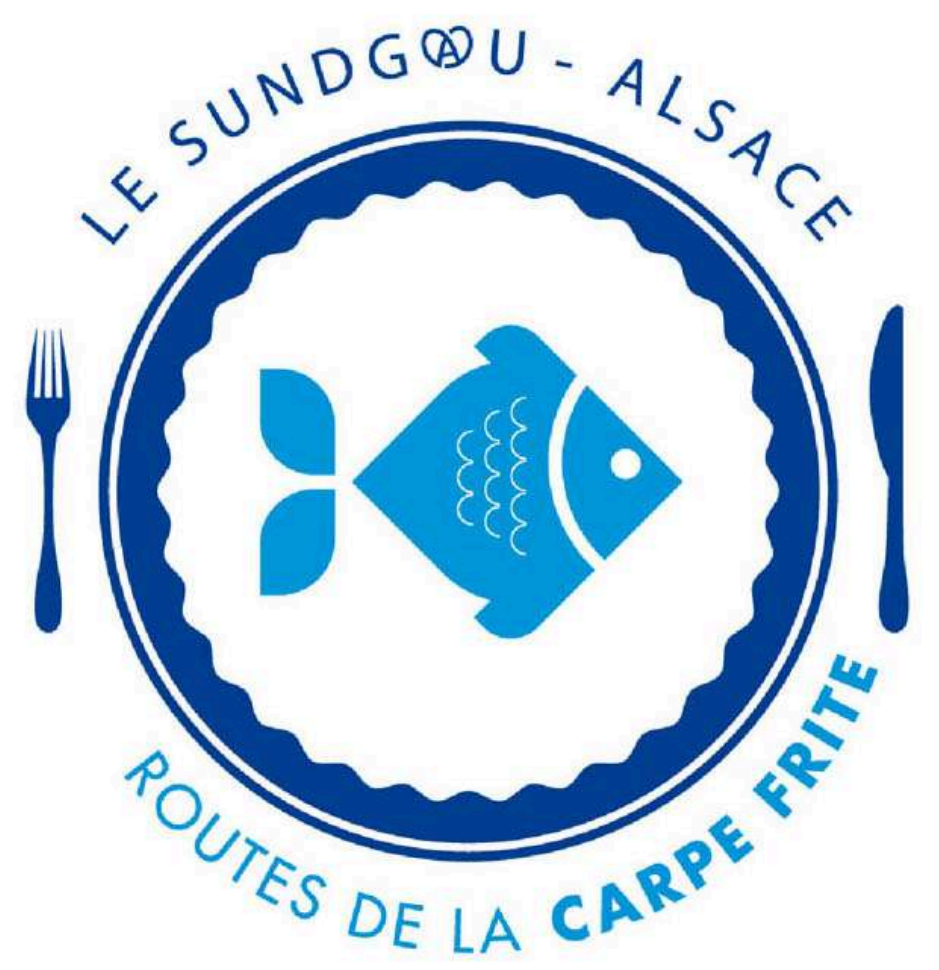

En 2015, le logo de la Route a été redessiné, en particulier pour y intégrer dans le "A » de «SUNDGAU » la forme de bretzel devenue la référence graphique de la politique de communication de la (défunte) Région Alsace (Figure 4). Au total, une vingtaine de restaurants sont membres de la Route et dix d'entre eux participent aux Carpailles 2016. Mais de fait, il est possible de se faire servir des carpes un peu partout ; la plupart des restaurateurs profitent de l'image Carpe frite sans adhérer à la Route, ce qui témoigne du primat de l'individualisme sur le sens collectif.

Dans le Sundgau, la pêche en eau libre est nécessairement gérée par une Association Agréée de Pêche et de Protection du Milieu Aquatique (AAPPMA). Au nombre de six, elles réglementent l'activité, délivrent les cartes de pêche et protègent le milieu aquatique. Hier comme aujourd'hui, la pêche des truites, chevennes et autres barbeaux en rivière garde la préférence des pêcheurs (Richert, 1991, cf. 14-15). La plupart des étangs échappent aux AAPPMA. Ils sont gérés par des propriétaires, privés, communaux ou associatifs qui y construisent de modestes installations (Figure 5). Usuellement, pour 10 euros par jour ou pour 50 euros par saison, la carpe, ce «poisson de sport par excellence » (Ranson, 2003), pourra être pêché ; parfois, l'étang est décrété no kill et il faudra rejeter l'animal à l'eau. Quant à la vente éventuelle de poisson par un pêcheur non-professionnel, elle est passible d'une amende de 3750 euros. 
Figure 5 : Un étang de pêche à Zillisheim. Route en impasse, gestion associative et clubhouse, panneau pédagogique, étang no kill et accès pour pêcheurs handicapés en fauteuil. RW 2016.

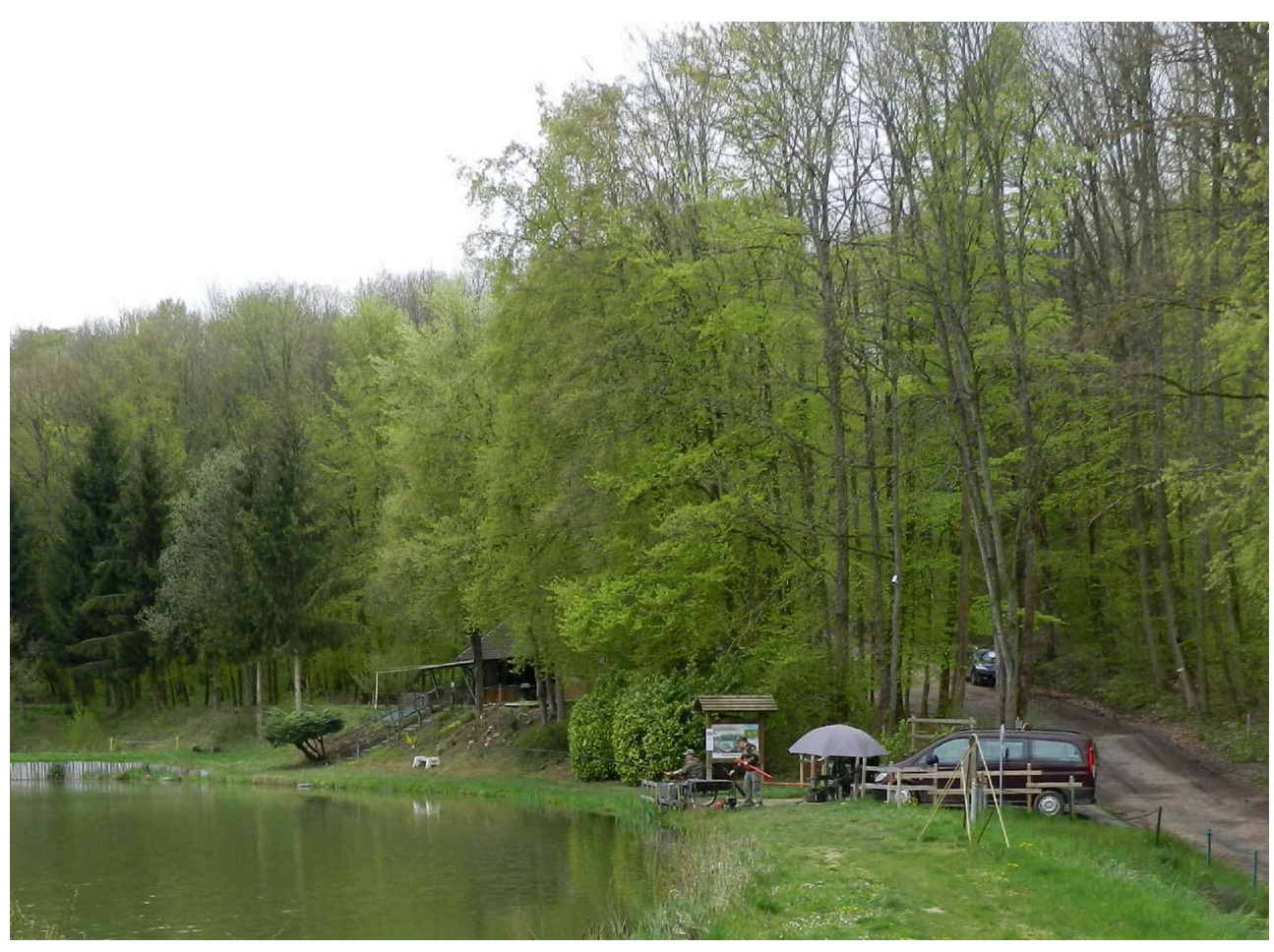

24 J. Haennig (1974) observe que le creusement des étangs avait connu un boom après 1945. D'une part, les engins de terrassements facilitaient enfin la tâche; d'autre part, des citadins, souvent héritiers d'un bien, y établissaient leur base de loisir. Ainsi, dans la conclusion du Bulletin de la Société Industrielle de Mulhouse de 1974, le Dr Michel Fernex imaginait des cohortes de pêcheurs qui passeraient leurs vacances in situ, renonçant ainsi aux grandes migrations touristiques responsables de pollutions et d'accidents de la route. Un rêve de militant écologiste, que l'on retrouve d'ailleurs à propos du Bourbonnais dans La soupe aux choux de René Fallet paru en 1974...

25 Faute de poids suffisant, aucune ville ne polarise vraiment le Sundgau, d'autant moins que, dans les petits centres, la rationalisation de l'administration leur a fait perdre de modestes mais précieuses fonctions. De même, l'agriculture productiviste et la désindustrialisation (tuileries, textile, constructions mécaniques...) ont conduit à des migrations pendulaires vers les centres extérieurs. C'est ainsi que depuis 1990, le Sundgau est éligible aux aides publiques et communautaires, à un moment où une tentative intéressante de projet territorial endogène émergeait avec Sundgau 2000, une association de Pays qui regroupait plus de 60.000 habitants avec 8 Établissements Publics de Coopération Intercommunale (EPCI) et 112 communes (Nonn, 2008) dont plusieurs dissidentes (Figure 6). Mais, dans les années 2000, le Pays s'était étiolé après l'extinction des fonds compensatoires à l'abandon du canal Rhin-Rhône à grand gabarit et avec l'arrivée des SCOT sur des périmètres plus petits. De fait, le Sundgau est une mosaïque de micro-territoires qui peinent à s'organiser, voire à se défendre. Les acteurs locaux ne pèsent guère par rapport aux services de l'État auxquels ils ne peuvent résister. Ainsi, Brice Martin (2005) souligne que, dans la vallée de la Largue, l'État a facilement imposé ses plans de prévention des risques Inondation et Mouvements de Terrain, qui ne représentent que des aléas faibles, alors que les coulées de boue sont un vrai sujet de préoccupation dont l'État n'a pas tenu compte. 


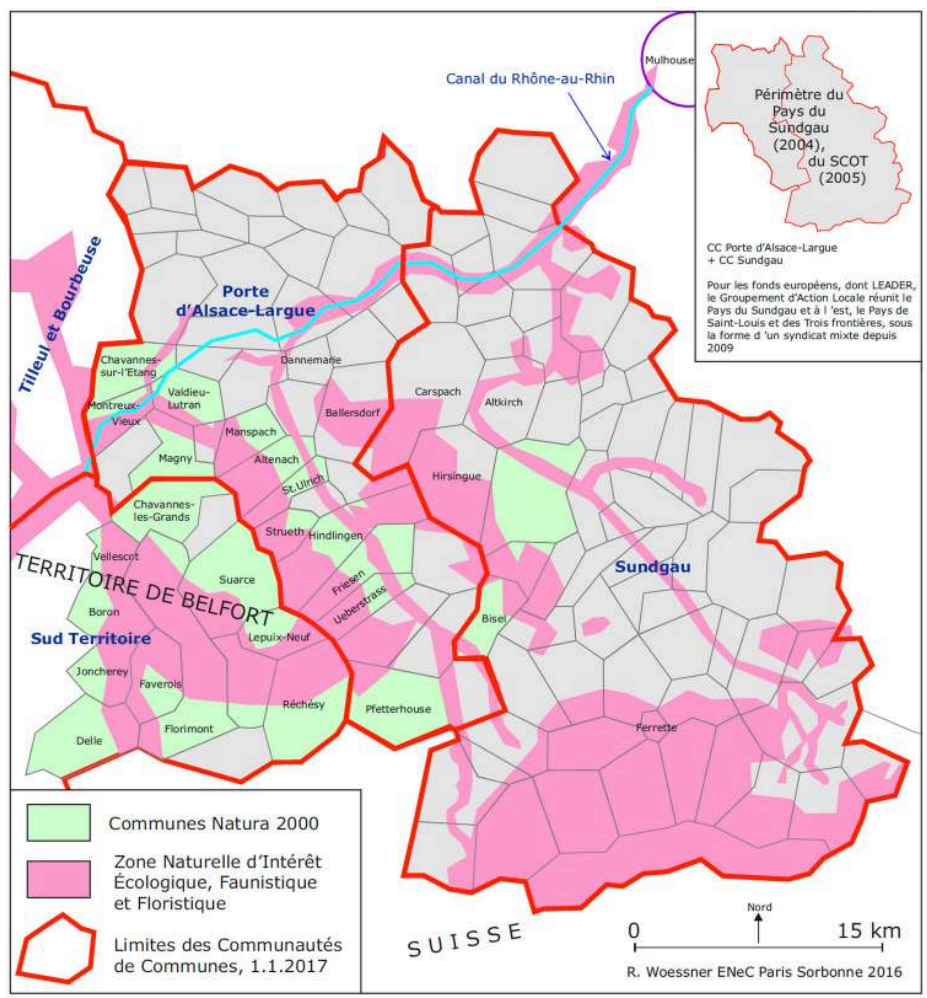

Par défaut, c'est la périurbanisation qui constitue le fait structurant, avec la construction de lotissements, voire de petits immeubles, et une économie présentielle non négligeable. La multipolarisation des communes sundgauviennes est inscrite dans l'histoire du $\mathrm{XX}^{\mathrm{e}}$ siècle: dans les Trente Glorieuses, «la quasi-totalité des jeunes cultivateurs [de Fulleren] s'étaient fait embaucher chez Peugeot à Sochaux, puis à Mulhouse, et certains d'entre eux pratiquaient la double activité de paysan et d'ouvrier. Les jeunes filles allaient travailler dans les filatures de Suisse, et des cars de ramassage assuraient aux uns et aux autres le transport du domicile à l'usine et vice-versa " (Zimmermann, 1982, cf. p.142). Peut-être la nouvelle Communauté de communes, qui voit revenir les 112 communes, et qui a été imposée par le préfet pour le $1^{\mathrm{er}}$ janvier 2017, va-t-elle permettre de relancer un projet partagé par l'ensemble du Sundgau. En effet, la loi NOTRe (Nouvelle Organisation Territoriale de la République) transfère progressivement des compétences aux EPCI comme la promotion du tourisme, l'aménagement des aires d'accueil des gens du voyage, la collecte et le traitement des déchets des ménages, l'eau et l'assainissement.

\section{Conclusion : entre-soi identitaire ou ouverture économique?}

Les pays d'étangs (Dombes, Saulnois, Pays des Étangs...) ont développé des initiatives avec des succès divers. Particularité du Sundgau, la route touristique et gastronomique a été construite autour d'un poisson-symbole, la carpe, alors même que ses pisciculteurs diversifient leurs activités par nécessité économique. La recette de la carpe frite témoigne d'une originalité qu'on ne retrouve pas sur les sites internet 
(Marmiton, par exemple). Il est vrai qu'elle n'est pas dans l'air du temps car elle s'adresse à de solides estomacs : la carpe est frite, les pommes de terre sont frites (alors que Suzanne Roth conseille des pommes vapeur, cf. Figure 3), on trempe le tout dans de la mayonnaise, on complète avec une salade verte, on arrose de vin blanc d'Alsace, riesling ou pinot, et un vrai Sundgauvien continuera avec un vacherin glacé ; pour les amateurs, le café-schnaps finira le tout avec bonheur. De fait, la Route s'adosse à un phénomène identitaire alors qu'aujourd'hui les préoccupations diététiques ont pris le pas sur les nécessités caloriques. D'où les Carpailles qui veulent précisément renouveler le genre.

Il reste à interroger la capacité des étangs à faire territoire. Le Sundgau est-il un «territoire limnique»? Bartout le définit comme « un territoire où les interrelations hommes-milieux-plans d'eau permettent d'identifier un espace aux caractéristiques limniques spatio-temporelles proches, offrant la possibilité, dans une optique de gestion, d'apporter une unité géographique, ceci à des échelles fluctuantes selon le facteur dominant envisagé " (Bartout, 2015). Or, la place prise par les étangs sundgauviens est discrète. Ils créent peu de valeur économique. Ils apparaissent comme des refuges discrets pour bien des familles et des pêcheurs. Jour de fête annuel du club de pêche excepté, le silence est dense autour des étangs. Il n'y a de comptes à rendre à personne, on se retire du monde, on échange des trucs de pêche, on communie avec une nature aménagée et un peu quelconque: des paysages ordinaires pour gens ordinaires, qui n'en demandent pas davantage.

Faut-il toucher à ces paysages et à ces pratiques qui témoignent d'une forme d'authenticité populaire ? Ou aller plus loin? Faut-il rester en marge ou tenter d'activer une mise en périphérie autre que la périurbanisation? (Figure 7). On entrerait alors dans une forme de limno-territorialisation, avec des étangs qui serviraient de lien fédérateur entre les acteurs du développement local, en vue d'un projet partagé en terme de développement durable. Le moment est peut-être favorable. 
Figure 7 : Forces centrifuges vs limno-territorialisation

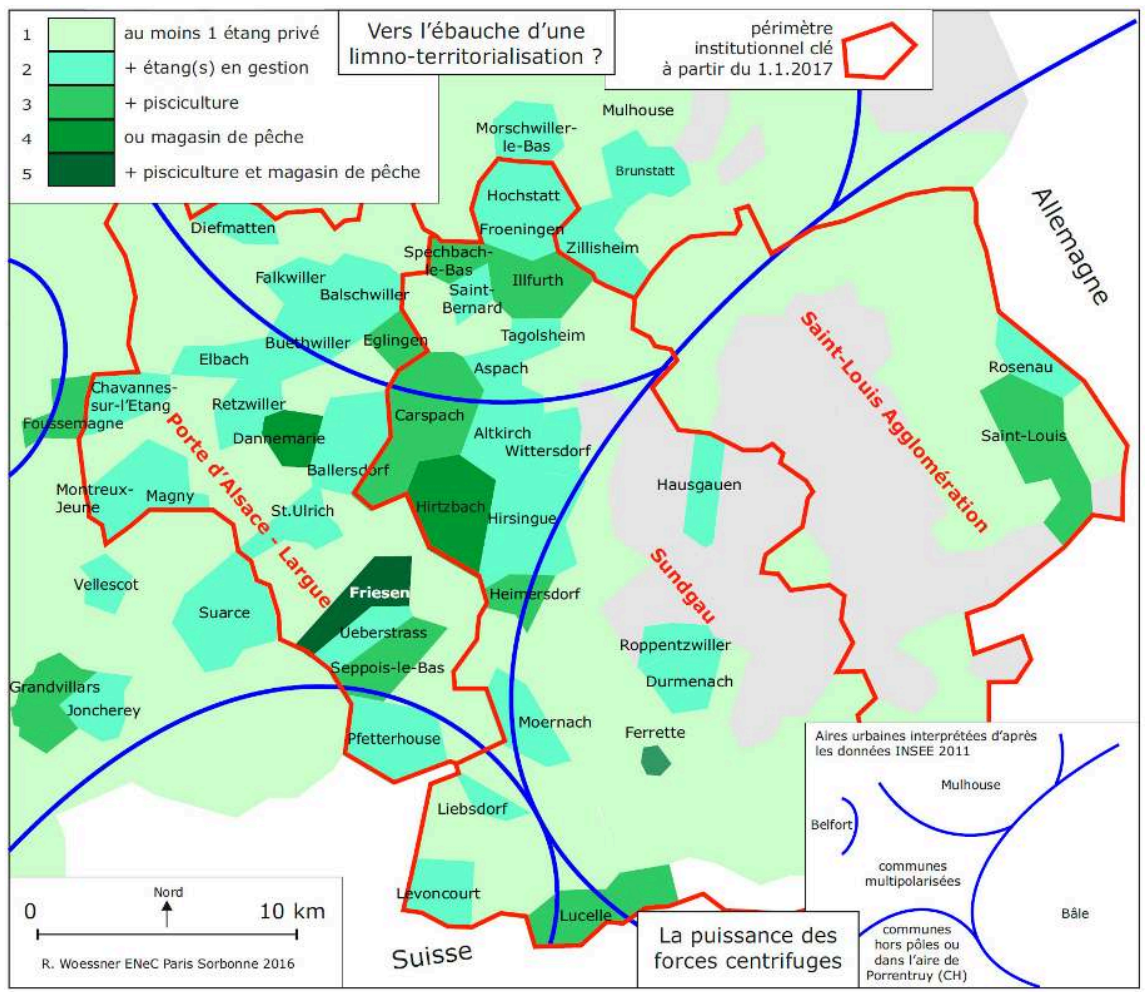

En effet, depuis 1975, les capacités systémiques du Sundgau s'étaient amoindries. Plusieurs facteurs y ont concouru: de manière générale, le Haut-Rhin est en mauvaise posture économique ; dans les nouvelles générations, beaucoup de jeunes gens qualifiés sont partis vivre ailleurs dans des métropoles, en France ou à l'étranger; la balkanisation administrative et politique a opposé les territoires les uns aux autres, parfois dans des dimensions minuscules; le phénomène de marge a généré des forces centripètes multiples, alors qu'il faudrait pouvoir compter sur des forces centrifuges.

En ce sens, la loi NOTRe offre des perspectives puisque des territoires de plus grande taille sont sur le point d'apparaître. Peut-être pourront-ils acquérir une dimension stratégique en entrant dans des logiques de projet. La création d'une intercommunalité sur la base du Pays du Sundgau ainsi que son association avec celui des Trois Frontières pour l'obtention partagée des fonds européens LEADER 2014-2020 vont dans ce sens. A contrario, la frontière administrative avec les communes du Territoire de Belfort semble forte.

Il faut souvent rejoindre un village sundgauvien par de petites routes, mais les portes d'entrée sont proches, avec les gares TGV de Belfort-Montbéliard, de Mulhouse et de Bâle, plus l'EuroAirport qui a franchi la barre des 7 millions de passagers en 2015. Il y aurait donc une politique agressive à mener en intégrant d'autres éléments du patrimoine dans les dispositifs. Par exemple, au moment de Noël, Altkirch propose des animations "gothiques » où les naïades et les sorcières sont de la partie. De même, la problématique des circuits courts, du bio et de l'équitable, de la monnaie locale (la Cigogne, en préparation à Mulhouse) commence à s'affirmer. Il faudrait donc trouver des articulations à la fois dans l'entre-soi sundgauvien tout comme avec les villes proches et lointaines. On peut se souvenir de la bonne réputation de l'établissement hydrothermal Kneipp de Carpasch, détruit pendant la Grande Guerre, où «de 
nombreux malades avaient trouvé la guérison par des bains réparateurs, et des hôtes en bonne santé un lieu d'amusement discret» (Walch, 2016, cf. p. 42). Ou plus récemment du projet Center Parcs prévu dans les confins sundgauviens, mais que le Territoire de Belfort a laissé échapper au profit de la Lorraine dans un site très comparable, non loin d'une autoroute, d'un canal Freycinet et d'un piémont de moyenne montagne.

Il reste à faire preuve de créativité en matière de jeu d'acteurs, et à trouver des clés permettant le développement durable en lieu et place du primat accordé à la conservation et à la préservation des étangs, qui ne pourront que rester en marge, voire s'étioler, faute de « limno-territorialisation ».

\section{BIBLIOGRAPHY}

Bartout P., 2006, Pour un référentiel des zones humides intérieures de milieu tempéré : l'exemple des étangs en Limousin (France), thèse de géographie, Université de Limoges, 498 p.

Bartout P., 2015, Les territoires limniques, nouveau concept limnologique pour une gestion géographique des milieux lentiques, HDR de géographie, Université d'Orléans, $444 \mathrm{p}$.

Bartout P., Touchart L., 2015, « La stabilité limnique dans le bassin de la Vienne. La place des plans d'eau au sein des milieux et des sociétés depuis le XIXe siècle », Norois 2015/1 n²34, consulté le 16 avril 2016, URL: https://norois.revues.org/5581.

Bavoux J.-J., Chapelon L., 2014, Dictionnaire d'analyse spatiale, Paris, A. Colin, 607 p.

Bischoff G., 2007, «Les blancs de la carpe. Pisciculture et pouvoir : l'exemple de l'Alsace autrichienne (14e-17e siècle) ", in Chauvard J.-F., Laboulais I. (dir.), Les fruits de la récolte, études offertes à Jean-Michel Boehler, Presses Universitaires de Strasbourg, 492 p., p. 179-195.

Bouleau G., Pont D., 2014, « Les conditions de référence de la directive cadre européenne sur l'eau face à la dynamique des hydrosystèmes et des usages ", Natures, sciences, sociétés. vol.22 $\mathrm{n}^{\circ} 1$. Consulté le 16 avril 2016. Publié en ligne : 2014-04-07 URL: http://dx.doi.org/10.1051/nss/ 2014016.

Candidature LEADER 2014-2020, non-daté, Pays du Sundgau et Pays de Saint-Louis et des Trois Frontières, $74 \mathrm{p}$.

Glotz M., 2006, Promenades historiques à travers le Sundgau occidental, Société d'Histoire du Sundgau, $223 \mathrm{p}$.

Haennig J., 1974, « Les étangs dans le Sundgau », Bulletin de la Société Industrielle de Mulhouse, cf. p. 117-118.

INRA , Commission Filière Poissons, 2007, Scénarios pour la pisciculture française en 2021, 24 p.

Kolar S., 1991, Les étangs dans le territoire de Belfort, mémoire de Maîtrise de géographie, Université de Franche-Comté, Besançon, 191p. + annexes.

Le Gléau J.-P., 1999, « Un zonage pourquoi faire? », projet d'article pour Les Annales des Pontset-Chaussées, 5p., cité par Laurent Couderchet et Xavier Amelot, « Faut-il brûler les Znieff? », 
Cybergeo : European Journal of Geography [En ligne], Espace, Société, Territoire, document 498, mis en ligne le 05 mai 2010, consulté le 28 février 2016. URL : http://cybergeo.revues.org/23052 ; DOI : $10.4000 /$ cybergeo.23052.

Martin B., 2005, « Risques et territoires ", Actes de la $5^{e}$ Table Ronde Rhin-Sud, APR, CRESAT, RECITS, 111p., cf. p. 61-73.

Natura 2000, 2015, FR4201811 - Sundgau, région des étangs, 11 p., consulté le 29 février 2016, URL: https://inpn.mnhn.fr/docs/natura2000/fsdpdf/FR4201811.pdf.

Nonn H., 2008, L'Alsace et ses territoires, Presses Universitaires de Strasbourg, 576 p.

ORTA, Observatoire Régional du Tourisme d'Alsace, consulté le 3 mars 2016, URL: http:// www.clicalsace.com/fr/cartographies.

Ranson S., 2003, L'alimentation de la carpe dans son biotope et en élevage, Thèse de médecine vétérinaire, Faculté de médecine de Créteil, $120 \mathrm{p}$.

Richert U., 1991, Retour au Sundgau, Strasbourg, La Nuée Bleue, 183 p.

Roth S., 1984, Les recettes de nos grands-mères du Sundgau, Steinbrunn-le-Haut, Éditions du Rhin, $141 \mathrm{p}$.

Schortanner M., 1978, « Les étangs », dans Collectif, Les eaux douces en Alsace, Wettolsheim, Mars et Mercure, $131 \mathrm{p}$.

Urban M.-P., 2010, La grande encyclopédie des lieux d'Alsace, Strasbourg, La Nuée Bleue, 558 p.

Walch A., 2016, 1914, un destin alsacien, Neuilly-sur-Seine, Atlande. 252 p.

Werner L.G., 1920, « Les étangs du Sundgau », Bulletin de la Société Industrielle de Mulhouse, cf. p. 176-177.

Woessner R., 2010, « Les conditions de la territorialisation », Revue d'Économie Régionale et Urbaine $\mathrm{n}^{\circ}$ 4-2010, Paris, Armand Colin, p. 669-687.

Zimermann A., 1982, Fulleren, un village du Sundgau, Bordeaux, Delmas,162 p.

\section{NOTES}

1. http://www.lyonpeople.com/news/p1amelienothomb.html, consulté le 5 mars 2016.

2. Emmanuel Claerr, 2010, http://www.crdp-strasbourg.fr/data/patrimoine-naturel/eau-01/ etangs.php?parent=16, consulté le 16 avril 2016.

3. http://alsace.lpo.fr/index.php/sundgau, consulté le 2 mars 2016.

4.

http://www.haut-rhin.gouv.fr/content/download/7944/45508/file/

Guide_ONEMA_Gestion_Patrimoniale.pdf, consulté le 16 avril 2016.

\section{ABSTRACTS}

Sundgau is a marginal region, located at the edge of the towns of the south Upper Rhine. Since 1975, the Fried Carp Road Association has been promoting this recipe, which has to do with a 
search of identity. Carp is not really a renowned fish for its taste. Therefore it looks hazardeous for a little rural region which wants to exist on the map. The ponds are here described, yesterday and today. The region is splitted in a lot of very small administrative and political units. Consequently, the merge of territorial forecasts and projects is improbable. Nevertheless, new forms of governance are on the table and new fields of activity could be done.

Le Sundgau est un espace de marge, quelque peu replié sur lui-même, et qui depuis 1975 promeut un plat identitaire avec l'association de la Route de la Carpe frite. La carpe elle-même n'est pas un poisson réputé pour ses qualités gustatives et, pour une petite région rurale peu connue, il est difficile d'exister sur la carte. Il s'agit de dresser un état des lieux autour de la question des étangs, d'examiner les compétences et les découpages de ce territoire, de voir quelles sont les fonctions dont il peut disposer. La balkanisation en micro-territoires a freiné l'élan initial, mais avec la constitution attendue d'entités administratives et politiques plus grandes, il n'est pas exclu que de nouvelles activités puissent se développer.

Der Sundgau ist ein peripherer Raum des südlichen Oberrheingebiets, etwas in sich selbst zurückgezogen, und in dem seit 1975 ein typisches Gericht durch den Verband der touristischen Straße des frittierten Karpfens gefördert wird. Für eine kleine wenig bekannte ländliche Gegend, ist es schwierig, auf der Karte zu existieren, weil der Karpfen selbst kein Fisch für seinen Geschmack bekannt ist. Dies ist eine Bestandsaufnahme, der Probleme rund um die Teiche aufzustellen, die Kompetenzbereiche und die Untergliederung des Territoriums zu untersuchen und die Funktionen zu sehen, die zur Verfügung stehen. Die Balkanisierung in Mikro Gebieten hat den Anfangsimpuls gedämpft, aber mit der erwarteten Einrichtung von größeren Verwaltungs- und politischen Einheiten, ist es nicht ausgeschlossen, neue Aktivitäten zu fördern.

\section{INDEX}

Mots-clés: étang, gastronomie, marge, patrimoine, paysage, prospective

Schlüsselwörter: Erbe, Gastronomie, Landschaft, Peripherie, Teich, Weitblick

Keywords: forecast, gastronomy, heritage, landscape, margin, pond 